\title{
MeV Neutrino Sources: The Sun and the Supernovae
}

\author{
Sylvaine Turck-Chièze \\ SAp/DAPNIA/DSM/CEA CE Saclay, 91191 Gif sur Yvette, France
}

\begin{abstract}
The two sources of $\mathrm{MeV}$ neutrinos, the Sun and the Supernovae are very interesting to study nowadays. Seismology aboard SoHO has properly constrained the solar emitted neutrino fluxes and consequently contributes to an unambiguous evidence of the solar neutrino oscillations. The main role of seismology stays nevertheless the description of the magneto-hydrodynamic processes and this progress provokes a renewal of the stellar discipline. Important results are noticed these three years, for the Sun. Future will be richer implying a large number of stars with an evident impact on supernovae and solar neutrino properties.
\end{abstract}

\section{The Two Sources of Neutrinos}

The two natural Neutrino MeV Sources, the Sun and the Supernovae, are studied with more and more interest as the richness of their plasma properties is inaccessible in laboratories. Nevertheless, the level of knowledge of these sources is extremely different. The solar internal structure is scrutinized for twenty years with two independent probes: neutrinos and acoustic modes. In the case of supernovae, the emitted neutrinos have been detected once only, for the $\mathrm{Su}$ pernova $1987 \mathrm{~A}$. It is evident that these two sources are extremely different. If the Sun has a continuous emission of neutrinos, the emission coming from a supernova explosion is quasi instantaneous, with a peak of $0.025 \mathrm{~s}$. Only 6.4 $\%$ of the produced solar energy is emitted by the neutrinos, though the main part of the released energy in a supernova explosion is contained in the neutrino emission. If the Sun emits neutrinos up to $14 \mathrm{MeV}$ and has a well determined energy spectrum, the range of emission for the supernovae is large from $5 \mathrm{MeV}$ to about $100 \mathrm{MeV}$. The impact of the different neutrino oscillation solutions on the supernova energy spectrum is high, mainly for the SNO detector, even the waiting statistics in Superkamiokande is higher, so there is extremely large interest to detect supernova neutrinos in the present observatories to put more constraints on the neutrino properties or on the explosion mechanism. The diversity of the supernovae (Heger et al. 2003) and consequently of the supernova explosions encourages also the study of the different supernova precursors. Supernovae Ia have initial mass below $8 \mathrm{M}_{\odot}$, and final mass probably below 1.5 $\mathrm{M}_{\odot}$, Supernovae II have initial mass between 8 to $30 \mathrm{M}_{\odot}$, final mass below 10 $\mathrm{M}_{\odot}$, Supernovae $\mathrm{Ib}$ and $\mathrm{c}$ have initial mass above $30 \mathrm{M}_{\odot}$ and final mass always below $10 \mathrm{M}_{\odot}$. With such a range in mass, questions on geometry, asphericity, rotation, metallicity have not a clear answer today.

The geometry of stars and the mass loss processes are more under control at different stages of evolution, so there is a renewal in stellar structure, which 
will benefit by seismic observations, capabilities of parallel computers and the introduction of macroscopic motions. The direct and indirect role of the rotation is now proven for the determination of the oblateness of massive stars. The impact on the geometry of the explosion is waiting. The role of the stellar metallicity is also a determinant factor, with cosmological consequences, it must be studied to interpret correctly the incoming large dataset supernova light curves.

We are convinced that a clear hydrostatic and hydrodynamic vision of stars will emerged within the next decade, thank to the spatial COROT and EDDINGTON projects with consequences for supernovae progenitors. Let summarize here the highlights of the last 3 years for the Sun which is the first star for which this effort is already well advanced.

\section{The Sun as a laboratory for emitted neutrino fluxes}

The Sun is a unique case for which we have today 40 years of observations: five neutrino experiments using different techniques, 3 helioseismic ground networks and 3 spatial helioseismic experiments aboard SoHO running since 7 years.

Since 1988, we have controlled the emitted solar neutrino fluxes, in confronting the solar theoretical structure to the seismic one. Using this probe as a reference, constant progress has been noticed on the determination of these fluxes (Turck-Chièze et al. 1988, Turck-Chièze and Lopes 1993, Dzitko et al. 1995, Brun, T-C \& Morel, 1998, Brun, T-C \& Zahn, 1999, Turck-Chièze et al. 2001a, Turck-Chièze et al. 2001b, Couvidat, Turck-Chièze \& Kosovichev 2003).

Helioseismology has contributed to stabilize the structure of the Sun thanks to the sound speed profile, helping to answer to questions on:

- 1) a proper determination of the metal opacity coefficients

- 2) a proper description of the microscopic diffusion

- 3) a proper description of the role of the plasma in nuclear reaction rates

- 4) the existence or not of a potential mixing in the very central core

- 5) the adequation of a Maxwellian distribution for particle velocities

- 6) the proper description of the $\mathrm{pp}$ reaction rate

- 7) the existence of mixing in the radiation-convection region partly inhibiting the microscopic diffusion.

It is because we have found answers to these questions that neutrino predictions coming from different groups have converged. If the two first questions have been solved with ground observations, the other ones, largely important for determining properly the neutrino fluxes, have needed the SoHO satellite. This is due to the nature of acoustic modes.

Excited by the superficial granulation, these modes are mainly sensitive to the convection zone more than the nuclear core. But the high energy neutrinos, those produced by the ppIII chain, the ${ }^{8} B$ neutrinos detected in Superkamiokande and SNO, are produced below $0.1 R_{\odot}$, it is also the case for the ${ }^{17} \mathrm{~F}$ and ${ }^{15} \mathrm{O}$ neutrinos. All these neutrinos are extremely dependent on the central temperature. But the extraction of the very internal structure from acoustic frequencies needs a great accuracy on the frequency determination. Unfortunately, for the easily detected global modes, the precision is degraded by stochastic excitation and variability with the solar cycle. The only way to escape 
this difficulty is to integrate the signal for a very long time, in order to reach acoustic modes of low frequency, less perturbed by these kind of processes.

Figure 1 of Couvidat, Turck-Chièze \& Kosovichev 2003, hereafter CTCK2003 , shows the improvement obtained on the quality of the data with the GOLF (Global Oscillations at Low Frequency, Gabriel et al. 1995) instrument aboard SoHO. This quality can be translated in an accuracy of $10^{-4}$ on the sound speed in the solar core (Turck-Chièze et al. 2001b). Such results put strong constraints and partial answers to questions 3 to 7 (Turck-Chièze et al. 2001a). Including progress on theoretical, instrumental and observational sides, we have finally produced seismic theoretical models. Nowadays, the number of emitted neutrino fluxes, and in particular the ${ }^{8} B$ neutrinos, is constrained by the helioseismic probe. A flux of $4.950 .7210^{6} \mathrm{~cm}^{-2} \mathrm{~s}^{-1}$ for the ${ }^{8} B$ neutrinos has been determined by Turck-Chièze et al. 2001b, with an error bar of only $14 \%$, including physics beyond standard hypotheses. The perfect agreement of this number with the results of the SNO detections is determinant to solve the neutrino puzzle, to demonstrate the solar oscillations and to put strong constraints on energy production including CNO cycle.

\section{Is the Sun standard or not?}

Helioseismology reveals that the Sun is not standard. The main objectives of this probe is to go beyond this simple representation which neglects the effects of the macroscopic motions. During these last 3 years, variabilities connected to the evolution during the solar cycle have been extracted and internal migrating flows have been observed. The history of the angular momentum evolution is crucial to understand the dynamo process and the range of internal magnetic field which is an essential property of the solar plasma.

Due to solar rotation and magnetic field, the frequencies of two modes of the same degree $\ell$ and order $\mathrm{n}$ are splitted in $\mathrm{m}$ components varying between $\pm \ell$. The corresponding splitting contains information on the internal rotation and presently one can extract this rotation profile down to the limit of the nuclear core, typically $0.2 \mathrm{R} \odot$, for the first time without ambiguity thanks to the longevity, the stability and the position of the SoHO satellite (Couvidat et al. 2003). It appears that the radiative zone rotates as a solid body with a constant rate but in examining gravity mode candidates, one cannot exclude a small core with a different axis and a quicker rotation, a release of the early rotation? (Couvidat et al. 2003).

The problem could be to see the impact of such phenomenon on the neutrino productions. We have concluded in CTCK2003 that it must be small (no more than $12 \%$ on the previous neutrino flux) with a limit on the magnetic field of $30 \mathrm{MG}$ in the radiative zone. Of course such assumptions must be confirmed by further works and probably a new generation of instruments that we are preparing. From the rotation profile, one begins to develop hydrodynamical calculations which have for objectives to reproduce the differential rotation in the convective zone (Brun \& Toomre, 2002). These hydrodynamic simulations may be useful to try to estimate some order of magnitude of the internal magnetic field to reproduce the solar magnetic cycle. Several works attack these problems. 


\section{The search for neutrino properties and Perspectives}

It is interesting now to study if some properties of the solar plasma may play a role on the neutrino propagation or transformation. It is why we (CTCK2003) have delivered the radial electron and neutron density profiles for our seismic model, together with some limits on the magnetic field and the magnetic neutrino moment: $\mu_{\nu}>510^{-15} \mu_{B}$ in the radiative zone and $\mu_{\nu}>310^{-12} \mu_{B}$ in the transition region between radiation and convection. We also give some probabilities on the RSFP transition for estimated magnetic field. We are also looking for effective variation of the magnetic field along the solar cycle in the external layers (Turck-Chièze, Nghiem and Piau 2003).

We are also trying to extract some effect in the neutrino data. Consequently, we have analyzed the Superkamiokande 10 days released data. In the research of periodicies, we note significant peaks around half the rotation of the Sun, and some others with a period around the rotation, but the spectrum is not unambiguous as it is the case of acoustic modes. We have also studied the correlation between the neutrino dataset and magnetic indicators. We note some correlation with a delay of several hundred days, which may translate some internal interaction. The same analysis has been repeated with SAGE data. But all the effects are not sufficiently significant.

I strongly recommend to deliver datasets of 5 days for Superkamiokande and SNO and to try to extract data in the low energy experiments at the same time, effectively, the magnetic activity evolves strongly at the scale of days.

\section{References}

Brun, A. S., Turck-Chièze, S., \& Morel, P. 1998, ApJ, 506, 913

Brun, A. S., Turck-Chièze, S., \& Zahn, J.P. 1999, ApJ, 525, 1032

Brun, A. S., Toomre, J. 2002, ApJ, 570, 865

Couvidat, S., Turck-Chièze, S, \& Kosovichev, A. 2003, ApJ, 599, 1434

Couvidat, S., Garcia, R.A., Turck-Chièze, S. Corbard, T., Henney, C. J., \& JiménezReyes, S. 2003, ApJ, 597, L77

Couvidat, S., Garcia, R. A., Turck-Chièze, S., Corbard, T. Henney, C. J., \& JimenezReyes, S. 2003, ApJ Lett., vol 597

Dzitko, H., Turck-Chièze, S., Delbourgo Salvador, P. \& Lagrange, C. 1995, ApJ, 447, 428

Gabriel, A., Grec, G., Charra, J., Robillot, JM., Roca Cortes, T., Turck-Chièze, S., et al. 1995, Sol. Phys., 162, 39

Heger, A., Fryer, C. L., Woosley, S. E., Langer, N., \& Hartmann, D. H. 2003, ApJ, 591, 288

Turck-Chièze, S., Cahen, S., Cass, M. \& Doom, C. 1988, ApJ, 335, 415

Turck-Chièze, S. \& Lopes, I. 1993, ApJ, 408, 347

Turck-Chièze, S., Nghiem, P. , Couvidat, S., and Turcotte, S. 2001a, Sol. Phys, 323

Turck-Chièze, S., et al. 2001b, ApJ 555, L69

Turck-Chièze, S., Nghiem, P., \& Piau, L. 2003, Highlights of Astronomy, 13, JD12 